\title{
Long wavelength perfect fluidity from short distance jet transport in quark-gluon plasmas
}

\author{
Jiechen $\mathrm{Xu}^{\mathrm{a}}$, Jinfeng Liao ${ }^{\mathrm{b}, \mathrm{c}}$, Miklos Gyulassy $^{\mathrm{a}}$ \\ ${ }^{a}$ Department of Physics, Columbia University, 538 West 120th Street, New York, NY, USA \\ ${ }^{b}$ Physics Department and CEEM, Indiana University, 2401 North Milo B. Sampson Lane, Bloomington, IN 47408, USA \\ ${ }^{c}$ RIKEN BNL Research Center, Building 510A, Brookhaven National Laboratory, Upton, NY 11973, USA
}

\begin{abstract}
We build a new phenomenological framework that bridges the long wavelength bulk viscous transport properties of the strongly-coupled quark-gluon plasma (sQGP) and short distance hard jet transport properties in the QGP. The full nonperturbative chromo-electric (E) and chromo-magnetic (M) structure of the near "perfect fluid" like sQGP in the critical transition region are integrated into a semi-Quark-Gluon-Monopole Plasma (sQGMP) model lattice-compatibly and implemented into the new CUJET3.0 jet quenching framework. All observables computed from CUJET3.0 are found to be consistent with available data at RHIC and LHC simultaneously. A quantitative connection between the shear viscosity and jet transport parameter is rigorously established within this framework. We deduce the $T=160-600$ $\mathrm{MeV}$ dependence of the QGP's $\eta / s$ : its near vanishing value in the near $T_{c}$ regime is determined by the composition of $\mathrm{E}$ and $\mathrm{M}$ charges, it increases as $T$ rises, and its high $T$ limit is fixed by color screening scales.
\end{abstract}

Keywords: Relativistic Heavy Ion Collisions, Jet Quenching, Perfect Fluidity, Quark-Gluon Plasmas

\section{Introduction}

To probe the fundamental properties of hot quark matter and the mechanism of color confinement through ultrarelativistic nucleus-nucleus collisions, it is necessary to consider both the perturbative and nonperturbative aspects of QCD carefully in heavy-ion phenomenology. Present quantitative analyses of the strongly-coupled quark-gluon plasma (sQGP) created in A+A reactions at RHIC and LHC [1] nevertheless divide in the two aspects: on the one hand, in the "soft" nonperturbative regime, the low transverse momentum $\left(p_{T}\right)$ long wavelength "perfect fluidity" of the sQGP is described by relativistic hydrodynamical simulations; on the other hand, in the "hard" regime, high $p_{T}$ short distance jet transport properties in the QGP computed from perturbative QCD (pQCD) models are compatible with a wide range of data [2]. A unified framework incorporating both aspects is however missing; it is therefore challenging to translate conveniently between heavy-ion and confinement physics.

Concentrated on pQCD, to build up such a framework, both the long and short distance transport properties of the QGP must be accounted for more systematically. In the "soft" sector, the "perfect fluid" like sQGP has a near vanishing shear viscosity to entropy density ratio $\eta / s=1 / 4 \pi$ bounded by quantum fluctuations [3, 4], however from leading order (LO) pQCD estimate, the QGP in the weakly-coupled limit (wQGP) has an $\eta / s \approx 0.071\left(\alpha_{s}^{2} \log \left(1 / \alpha_{s}\right)\right)^{-1}$ that approaches 1 [5]. In the "hard" sector, it has been found 
that most jet energy loss models can describe the high $p_{T}$ light hadrons' and open heavy flavors' nuclear modification factor $\left(R_{A A}\right)$ data, but the azimuthal elliptic anisotropy $\left(v_{2}\right)$ is underestimated by $50 \%$ at RHIC and LHC near-universally [8].

The above necessitates (1) exploring the full nonperturbative chromo-electric (E) and chromo-magnetic (M) structure of QCD in the region near the critical transition temperature $\left(T_{c}\right),(2)$ developing a microscopic, lattice-compatible description of the sQGP, and (3) implementing it into a systematic pQCD jet energy loss model and testing with high $p_{T}$ data. The new CUJET3.0 framework achieved all of them [9].

\section{The CUJET3.0 framework}

In CUJET3.0 [9], accounting for both chromo-electric $(E)$ and chromo-magnetic $(M)$ quasi-particles (QPs) as in the EM seesaw scenario proposed by Liao and Shuryak [10], the dynamical running coupling DGLV [11] energy loss kernel in CUJET2.0 [12] is generalized to:

$$
x \frac{d N}{d x} \propto \int d^{2} q\left[\frac{\rho \alpha_{s}^{2}\left(\mathbf{q}_{\perp}^{2}\right) f_{E}^{2}}{\mathbf{q}_{\perp}^{2}\left(\mathbf{q}_{\perp}^{2}+f_{E}^{2} \mu^{2}\right)}\right] \ldots \rightarrow \int d^{2} q\left[\frac{\rho_{E}\left(\alpha_{s}\left(\mathbf{q}_{\perp}^{2}\right) \alpha_{s}\left(\mathbf{q}_{\perp}^{2}\right)\right) f_{E}^{2}}{\mathbf{q}_{\perp}^{2}\left(\mathbf{q}_{\perp}^{2}+f_{E}^{2} \mu^{2}\right)}+\frac{\rho_{M}\left(\alpha_{E}\left(\mathbf{q}_{\perp}^{2}\right) \alpha_{M}\left(\mathbf{q}_{\perp}^{2}\right)\right) f_{M}^{2}}{\mathbf{q}_{\perp}^{2}\left(\mathbf{q}_{\perp}^{2}+f_{M}^{2} \mu^{2}\right)}\right] \ldots
$$

Here $\alpha_{s}\left(Q^{2}\right) \equiv \alpha_{E}\left(Q^{2}\right)=\alpha_{c} /\left[1+\frac{9 \alpha_{c}}{4 \pi} \log \left(\frac{Q^{2}}{T_{c}^{2}}\right) \cdot 1_{Q>T_{c}}\right], T_{c}=160 \mathrm{MeV}$, and $\alpha_{E} \cdot \alpha_{M}=1$ for any $Q^{2}$ because of Dirac quantization [10]. The total quasi-particle number density $\rho$ consists of EQPs with fraction $\chi_{T}=\rho_{E} / \rho$ and MQPs with fraction $1-\chi_{T}=\rho_{M} / \rho$. The color electric charges are suppressed near $T_{c}$ as in the semiQGP model [13], $\chi_{T} \equiv \chi_{T}^{L}=c_{q} L+c_{g} L^{2}$, where the Polyakov loop $L(T) \propto\left\langle\operatorname{tr} \mathcal{P} \exp \left\{i g \int_{0}^{1 / T} A_{0} d \tau\right\}\right\rangle$ is renormalized such that $L(T \rightarrow \infty)=1, c_{q}$ and $c_{g}$ are Stefan-Boltzmann fraction coefficients. In the critical transition region, the semi-QGP degrees of freedom (DOFs) and emergent chromo-magnetic monopoles form a semi-Quark-Gluon-Monopole Plasma (sQGMP) [9] 14]. The parameter $f_{E}$ and $f_{M}$ is defined via $f_{E} \equiv \mu_{E} / \mu=\sqrt{\chi_{T}}$ and $f_{M} \equiv \mu_{M} / \mu=c_{m} g$, where $\mu_{E}$ and $\mu_{M}$ is the E and M screening mass respectively, and $g=\sqrt{4 \pi \alpha_{s}\left(\mu^{2}\right)}=\mu /\left(T \sqrt{1+N_{f} / 6}\right)$.

The $L(T), \mu_{E, M}(T), \rho / T^{3} \sim p / T^{4}=\frac{1}{V T^{3}} \log Z$, and equation of state (EOS) are all constrained by lattice QCD data, as shown in Fig. 1. A theoretical uncertainty in CUJET3.0 is originated from choosing the diagonal u-quark number susceptibility $\chi_{2}^{u}(T)=\frac{\partial^{2}\left(p / T^{4}\right)}{\partial\left(\mu_{u} / T\right)^{2}}=\frac{1}{V T^{3}}\left\langle N_{u}^{2}\right\rangle$ over the Polyakov loop for the quark deconfinement rate, i.e. $\chi_{T} \rightarrow \chi_{T}^{u}=c_{q} \chi_{2}^{u}(T) / \chi_{2}^{u}(\infty)+c_{g} L^{2}$, which will be analyzed lately. All other computational details in CUJET3.0 are the same as in CUJET2.0, including the $2+1 \mathrm{D}$ viscous hydrodynamical background profiles generated from VISHNU simulations [15].
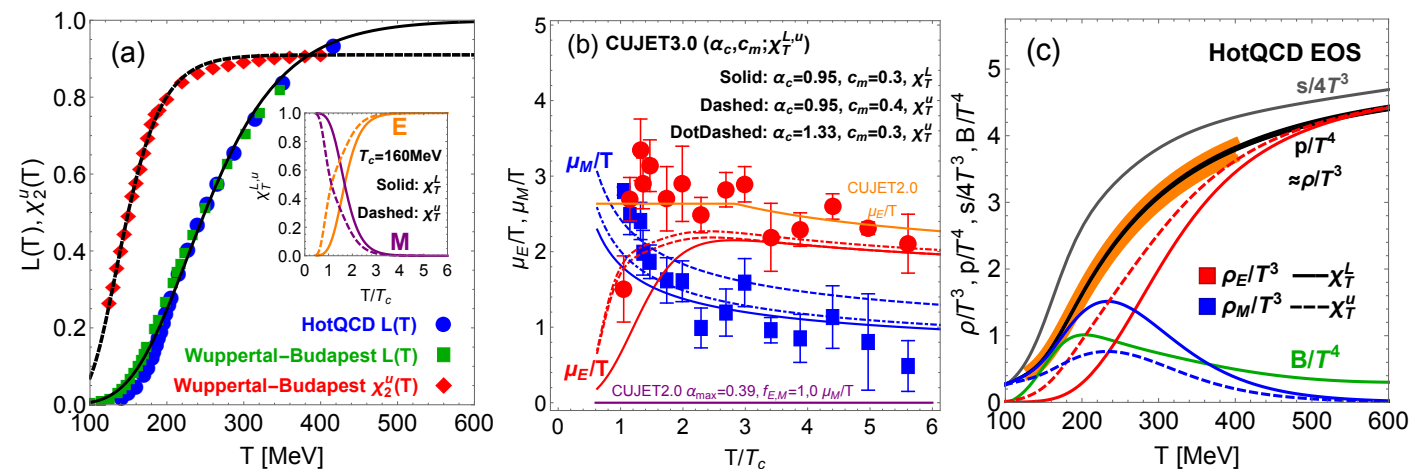

Fig. 1. (Color online) (a) The parameterized fit to lattice QCD data [6] of the renormalized Polykov loop $L$ and diagonal light quark susceptibility $\chi_{2}^{u}$ in the $\chi_{T}^{L}$ and $\chi_{T}^{u}$ scheme within CUJET3.0. The inset shows the chromo-electric $(E)$ and chromo-magnetic $(M)$ quasiparticle fractions in corresponding schemes. (b) The temperature dependence of the $E$ and $M$ screening mass $\mu_{E, M}$ in CUJET2.0 (HTL QGP) and CUJET3.0 (sQGMP) compare with lattice simulations [7]. (c) The HotQCD equation of state (EOS, pressure $p$, entropy density s) [6], the "bag" pressure ( $B$ ), as well as the $E$ and $M$ quanta number density $\rho_{E, M}$ embedded in the CUJET3.0 framework. 

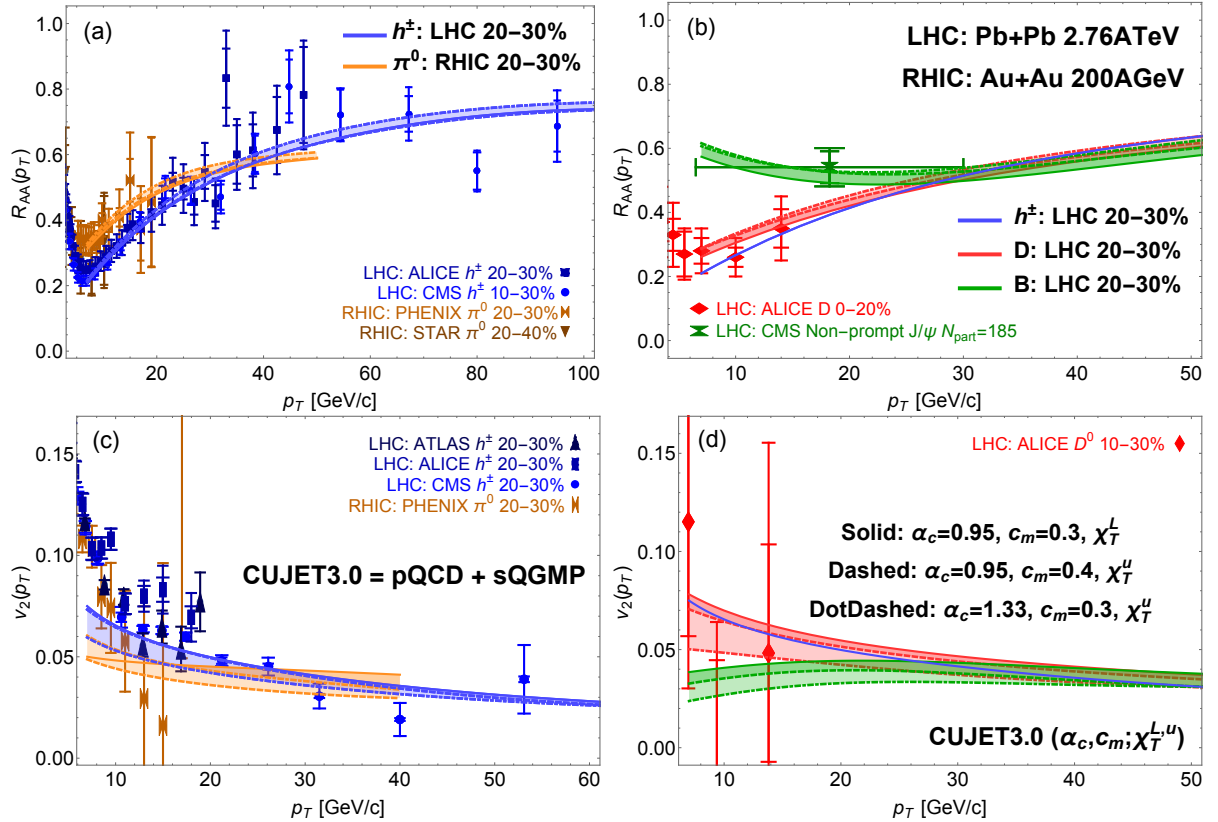

Fig. 2. (Color online) CUJET3.0 results of (a) light hadron (LH, neutral pion $\pi^{0}$ and charge particle $h^{ \pm}$)'s $R_{A A}$, (b) open heavy flavor (HF, $B$ meson and prompt $D$ meson)'s $R_{A A}$, (c) LH's $v_{2}$, and (d) HF's $v_{2}$, at high $p_{T}>8 \mathrm{GeV}$ in semi-peripheral A+A collisions, compared with data from RHIC and LHC [2]. The variations of predicted jet quenching observables from different schemes within CUJET3.0 suggest that data on high $p_{T}$ leading hadron $R_{A A}$ and $v_{2}$ in heavy-ion collisions can rigorously constrain the nonperturbative chromo-electric and chromo-magnetic structure of the QCD matter near $T_{c}$, and provide critical information about color confinement.

\section{Results and discussions}

Jet quenching observables from three different schemes in the CUJET3.0 framework will be studied: (i) $\alpha_{c}=0.95, c_{m}=0.3, \chi_{T}^{L}$; (ii) $\alpha_{c}=0.95, c_{m}=0.4, \chi_{T}^{u}$; (iii) $\alpha_{c}=1.33, c_{m}=0.3, \chi_{T}^{u}$. The parameter set $\left(\alpha_{c}, c_{m}\right)$ is constrained by the reference datum at LHC $20-30 \% \mathrm{~Pb}+\mathrm{Pb} \sqrt{s_{N N}}=2.76 \mathrm{TeV} R_{A A}^{h^{ \pm}}\left(p_{T}=12.5 \mathrm{GeV}\right) \approx 0.3$ and lattice date of $\mu_{E, M}(T)$ as shown in Fig. 1 (b). Fig. 2 compares the CUJET3.0 results of leading light hadron (LH) and open heavy flavor (HF)'s $R_{A A}\left(p_{T}>8 \mathrm{GeV}\right)$ and $v_{2}\left(p_{T}>8 \mathrm{GeV}\right)$ at RHIC and LHC semiperipheral $\mathrm{A}+\mathrm{A}$ collisions with corresponding data.

For high $p_{T}$ LHs, all three schemes can simultaneously describe the $R_{A A}$ and $v_{2}$ data at RHIC and LHC. The phenomenon that scheme (i) and (ii) generate a relatively larger $v_{2}$ than scheme (iii) implies that the azimuthal asymmetry is sensitive to how the relative value of $\mu_{E}$ and $\mu_{M}$ inverses near $T_{c}$ - the higher the inversion temperature, the longer the path length that jets interact with the monopole dominated medium at later time of the QGP evolution, the larger the high $p_{T} v_{2}$.

For open heavy flavors, scheme (ii) and (iii)'s $R_{A A}$ overlap, both are larger than scheme (i)'s. Since the former two have the same color deconfinement scheme $\chi_{T}^{u}$ that is different from the latter's $\chi_{T}^{L}$, it is implicit that the HF's high $p_{T} R_{A A}$ in CUJET3.0 is sensitive to the rate at which electric DOFs are liberated $\left(r_{d}=d \chi_{T} / d T\right)$, i.e. the detailed composition of $\mathrm{E}$ and M DOFs near $T_{c}$. Meanwhile, Fig. 2(d) shows that the HFs' $v_{2}$ 's are all different in scheme (i)(ii)(iii). It is therefore fair to conclude that the open charm and beauty's $R_{A A}\left(p_{T}\right)$ and $v_{2}\left(p_{T}\right)$ are excellent probes of the nonperturbative $\mathrm{E}$ and $\mathrm{M}$ structure of the sQGMP $\left(r_{d}, \mu_{E}, \mu_{M}\right)$ near $T_{c}$ within CUJET3.0.

The jet transport parameter $\hat{q}(T, E) \equiv\left\langle q_{\perp}^{2}\right\rangle / \lambda$ in CUJET3.0 and CUJET2.0 can be extracted as in [9] and [12, 17] respectively. They are plotted in Fig. 3 (a). Extrapolated $\hat{q}(T, E)$ down to thermal energy scales $E \sim$ $3 T$, one can estimate the $\eta / s$ using kinetic theory, i.e. $\eta / s=\frac{1}{s} \frac{4}{15} \sum_{a} \rho_{a}\langle p\rangle_{a} \lambda_{a}^{\perp}=\frac{18 T^{3}}{5 s} \sum_{a} \rho_{a} / \hat{q}_{a}(T, E=3 T)$, where $\rho_{a}(T)$ is the quasi-parton number density of type $a=q, g, m$. The $\eta / s$ results from both CUJET3.0 and CUJET2.0 are shown in Fig. 3 b). 

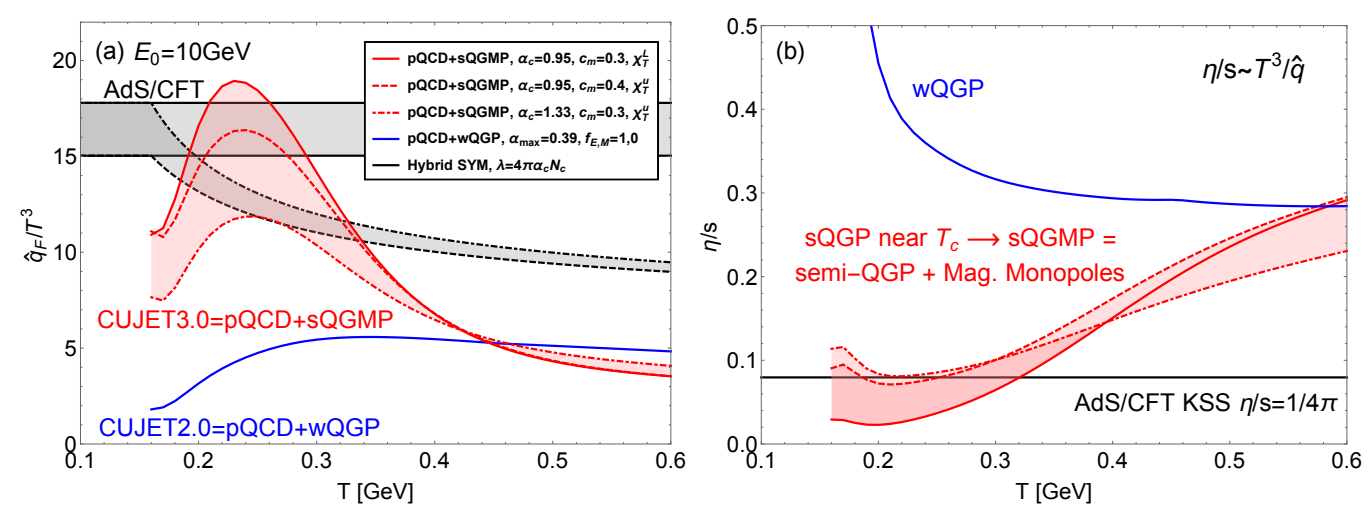

Fig. 3. (Color online) (a) The temperature dependence of the scaled jet transport parameter $\hat{q} / T^{3}$ for a quark jet (in the fundamental representation $F$ of $\mathrm{SU}\left(N_{c}=3\right)$ ) with initial energy $E_{0}=10 \mathrm{GeV}$ in various schemes within the CUJET3.0 framework, compared with the CUJET2.0 counterpart, as well as $\mathcal{N}=4$ Supersymmetric Yang-Mills (SYM) $\hat{q}_{\mathrm{SYM}}$ results from leading order (LO) AdS/CFT calculations $\left(\hat{q}_{\mathrm{SYM}}=\left[\pi^{3 / 2} \Gamma(3 / 4) / \Gamma(5 / 4)\right] \sqrt{\lambda} T_{\mathrm{SYM}}^{3}\right)\left[16\right.$. Note that $3 T_{\mathrm{SYM}}^{3} \approx T^{3}$ because of different number of degrees of freedom in $N_{c}=3 \mathrm{SYM}$ and three-flavor QCD [17]. The gray band with dashed black edges corresponds to using 't Hooft $\operatorname{coupling} \lambda=12 \pi \alpha_{s}\left(Q^{2}\right)$. (b) The shear viscosity to entropy density ratio $\eta / s$ estimated in the kinetic theory extrapolation $\eta / s \sim T^{3} / \hat{q}$ from jet quenching parameters in panel (a). Note that $T_{c}=160 \mathrm{MeV}$. In CUJET3.0, a clear $\hat{q}_{F} / T^{3}$ maximum and $\eta / s$ minimum appear at $T \sim 1.3-1.4 T_{c}$ where the scaled number density of emergent chromo-magnetic monopoles near $T_{c}$ peaks. The $(\eta / s)_{\min }$ is influenced by fractions of $\mathrm{E}$ and $\mathrm{M}$ composites, hence is sensitive to confinement physics. Its value in both $\chi_{T}^{L, u}$ schemes converge to approximately the KSS quantum bound $\eta / s=1 / 4 \pi$ [4]. At high $T$, the $\eta / s$ from sQGMP and weakly-coupled QGP (wQGP) coincide because of similar color screening structures.

At high $T$ where MQPs vanish as $\chi_{T} \rightarrow 1$, the $\eta / s$ of sQGMP and weakly-coupled QGP (wQGP) overlap because of similar $\mu_{E}(T)$ 's. In CUJET3.0, through fixing $\eta / s \sim T^{3} / \hat{q}$ at all temperatures, as $T$ cools down, $\eta / s$ drops, and a shear viscosity minimum appears at $T \sim 1.3-1.4 T_{c}$, coinciding with the temperature where $\rho_{M} / T^{3}$ peaks as shown in Fig. 1 1 (c). The value of $(\eta / s)_{\min }$ is determined by the deconfinement scheme $\chi_{T}^{L, u}$, i.e. EQP and MQP fractions near $T_{c}$, and it approaches the KSS quantum bound $\eta / s=1 / 4 \pi$ [4]. These indicate within the CUJET3.0 framework, the long wavelength "perfect fluidity" of the sQGP is generated from short distance jet transport properties controlled by $\hat{q}$, and a quantitative $\eta / s \sim T^{3} / \hat{q}$ connection is robustly established in a wide temperature range.

\section{Summary}

We conclude that taking full advantage of the new CUJET3.0 jet energy loss framework, data of high $p_{T}$ light hadron (LH) and open heavy flavor (HF)'s $R_{A A}$ and $v_{2}$ in heavy-ion collisions at RHIC and LHC can provide stringent constraints on the nonperturbative properties of the QCD matter near $T_{c}$. After fixed model parameters with LH's $R_{A A}$ data, (1) LH's $v_{2}$ regulates the E and M screening mass difference $\left(\mu_{E}(T)-\right.$ $\left.\mu_{M}(T)\right)$ near $T_{c}$, (2) HF's $R_{A A}$ determines the rate at which color DOFs are deconfined $\left(r_{d}(T)\right)$, (3) HF's $v_{2}$ distinguishes $r_{d}(T), \mu_{E}(T)$ and $\mu_{M}(T)$.

In the CUJET3.0 framework, after included the semi-QGP suppression of chromo-electric charges and the emergence of chromo-magnetic monopoles in the nonperturbative near-critical QGP, the long wavelength "perfect fluidity" $(\eta / s \sim 1 / 4 \pi)$ is successfully generated from the short distance hard parton transport properties that are controlled by the jet quenching parameter $\hat{q}$. Within this framework, a robust $\eta / s \sim T^{3} / \hat{q}$ connection is established in all temperature ranges above $T_{c}$. Overall, CUJET3.0 provides a quantitative bridge between heavy-ion phenomenology and fundamental confinement physics.

We thank Peter Petreczky for insightful discussions. JX acknowledges helpful conversations with Gabriel Denicol, Rob Pisarski, Chun Shen and Xin-Nian Wang. The research of JX and MG is supported by U.S. DOE Nuclear Science Grants No. DE-FG02-93ER40764. The research of JL is supported by the National Science Foundation (Grant No. PHY-1352368). JL also acknowledges partial support from the RIKEN BNL Research Center. 


\section{References}

[1] M. Gyulassy and L. McLerran, Nucl. Phys. A 750, 30 (2005).

[2] B. Abelev et al. [ALICE Collaboration], Phys. Lett. B 719, 18 (2013); Phys. Lett. B 720, 52 (2013); G. Aad et al. [ATLAS Collaboration], Phys. Lett. B 707, 330 (2012); S. Chatrchyan et al. [CMS Collaboration], Eur. Phys. J. C 72, 1945 (2012); Phys. Rev. Lett. 109, 022301 (2012); A. Adare et al. [PHENIX Collaboration], Phys. Rev. Lett. 101, 232301 (2008); 105, 142301 (2010); Phys. Rev. C 87, 034911 (2013); B. I. Abelev et al. [STAR Collaboration], Phys. Rev. C 80, 044905 (2009).

[3] P. Danielewicz and M. Gyulassy, Phys. Rev. D 31, 53 (1985).

[4] P. Kovtun, D. T. Son and A. O. Starinets, Phys. Rev. Lett. 94, 111601 (2005).

[5] T. Hirano and M. Gyulassy, Nucl. Phys. A 769, 71 (2006).

[6] A. Bazavov et al., Phys. Rev. D 80, 014504 (2009); S. Borsanyi et al. [Wuppertal-Budapest Collaboration], JHEP 1009, 073 (2010); S. Borsanyi, Z. Fodor, S. D. Katz, S. Krieg, C. Ratti and K. Szabo, JHEP 1201, 138 (2012).

[7] A. Nakamura, T. Saito and S. Sakai, Phys. Rev. D 69, 014506 (2004).

[8] B. B. Abelev et al. [ALICE Collaboration], Phys. Rev. C 90, no. 3, 034904 (2014); S. Cao, G. Y. Qin and S. A. Bass, Phys. Rev. C 92, 024907 (2015); B. Betz and M. Gyulassy, JHEP 1408, 090 (2014) [JHEP 1410, 043 (2014)]; arXiv:1503.07671] [hep-ph]; D. Molnar and D. Sun, arXiv:1305.1046 [nucl-th].

[9] J. Xu, J. Liao and M. Gyulassy, Chin. Phys. Lett. 32, 092501 (2015); arXiv:1508.00552 [hep-ph].

[10] J. Liao and E. Shuryak, Phys. Rev. C 75, 054907 (2007); Phys. Rev. Lett. 101, 162302 (2008); 102, 202302 (2009).

[11] M. Gyulassy et al., Nucl. Phys. B 594, 371 (2001); M. Djordjevic et al., Nucl. Phys. A 733, 265 (2004); Phys. Rev. Lett. 101, 022302 (2008); S. Wicks et al., Nucl. Phys. A 784, 426 (2007); A. Buzzatti et al., Phys. Rev. Lett. 108, 022301 (2012).

[12] J. Xu, A. Buzzatti and M. Gyulassy, JHEP 1408, 063 (2014); Nucl. Phys. A 932, 128 (2014).

[13] R. D. Pisarski, Phys. Rev. D 74, 121703 (2006); Y. Hidaka and R. D. Pisarski, Phys. Rev. D 78, 071501 (2008); 81, 076002 (2010); A. Dumitru et al., 86, 105017 (2012); C. Gale et al., Phys. Rev. Lett. 114, 072301 (2015).

[14] B. G. Zakharov, JETP Lett. 101, 587 (2015); A. Iwazaki, arXiv:1511.02271 [hep-ph].

[15] H. Song and U. W. Heinz, Phys. Rev. C 78, 024902 (2008); C. Shen, U. Heinz, P. Huovinen and H. Song, Phys. Rev. C 82, 054904 (2010); C. Shen, Z. Qiu, H. Song, J. Bernhard, S. Bass and U. Heinz, Comput. Phys. Commun. 199, 61 (2016).

[16] H. Liu, K. Rajagopal and U. A. Wiedemann, Phys. Rev. Lett. 97, 182301 (2006).

[17] K. M. Burke et al. [JET Collaboration], Phys. Rev. C 90, 014909 (2014). 